\title{
Condensation in Temporally Correlated Zero-Range Dynamics
}

\author{
Ori Hirschberg, ${ }^{1}$ David Mukamel, ${ }^{1}$ and Gunter M. Schütz ${ }^{2}$ \\ ${ }^{1}$ Department of Physics of Complex Systems, Weizmann Institute of Science, 76100 Rehovot, Israel \\ ${ }^{2}$ Institut für Festkörperforschung, Forschungszentrum Jülich, 52425 Jülich, Germany
}

(Received 4 June 2009; revised manuscript received 27 July 2009; published 26 August 2009)

\begin{abstract}
The impact of temporally correlated dynamics on nonequilibrium condensation is studied using a nonMarkovian zero-range process (ZRP). We find that memory effects can modify the condensation scenario significantly: (i) For mean-field dynamics, the steady state corresponds to that of a Markovian ZRP, but with modified hopping rates which can affect condensation; (ii) for nearest-neighbor hopping dynamics in one dimension, the condensate is found to occupy two adjacent lattice sites and to drift with a finite velocity. The validity of these results in a more general context is discussed.
\end{abstract}

DOI: 10.1103/PhysRevLett.103.090602

Many nonequilibrium systems are known to exhibit realspace condensation in which a high density phase condenses into a small region in space. Examples include phase separation in driven diffusive systems [1,2], jamming in traffic flow [3,4], gelation in networks resulting in a hub with a macroscopic linking number [5,6]. A similar phenomenon may occur in the clustering of compartmentalized shaken granular gasses. Under certain conditions condensation is observed, whereby most particles accumulate in one compartment $[7,8]$.

A paradigmatic model to study condensation in such nonequilibrium systems is the zero-range process (ZRP) $[9,10]$. In this model of mass transfer, particles hop between boxes with hopping rates $u(n)$ which depend only on the occupation number $n$ of the departing box. The steadystate distribution function of the box occupation numbers factorizes into a product of single-box terms, which makes this model amenable to theoretical studies. For rates which for large $n$ are of the form

$$
u(n)=\gamma(1+b / n),
$$

it has been shown that the model exhibits a condensation transition at high densities as long as $b>2$. At densities above the condensation transition, one of the boxes is occupied by a macroscopic number of particles, constituting a real-space condensate. The parameter $\gamma$ sets the time scale of the process. By mapping the dynamics of a specific physical system to that of a ZRP, one can study condensation phenomena in that system.

In reality, mass transfer processes usually constitute a coarse-grained description of more complex microscopic dynamics. This frequently results in temporally correlated dynamical processes, which are not captured in the Markovian dynamics of the ZRP [11]. It would thus be of interest to explore the effect of temporal correlations on the collective behavior of the ZRP, particularly on the occurrence of condensation.

In this Letter, we consider a non-Markovian ZRP, where the hopping processes depend not only on the present
PACS numbers: 05.70.Ln, 02.50.Ey, 05.40. $-\mathrm{a}, 64.60 .-\mathrm{i}$

occupation of a site but also on jump events that have occurred in the past. We find that this has significant impact on condensation. Specifically, two main results are derived. The first is that memory effects on steady-state condensation can be captured by a Markovian ZRP with an effective hopping parameter $b_{\text {eff }} \neq b$ which controls the condensation transition. The second effect is that in the case of asymmetric nearest-neighbor hopping on a ring of $L$ sites, the condensate drifts with a finite velocity which scales as $1 / L$. These results are based on studies of a particular temporally correlated dynamics, but we expect them to be rather generic.

The non-Markovian ZRP introduced in this work consists of $L$ boxes $i$, each characterized by the particle number $n_{i}$ and an internal "clock" $\tau_{i}$. The total number of particles is $N$. The clock proceeds irregularly in integer steps and is reset to zero each time a particle jumps onto site $i$, thus keeping a memory of the history of the process. A configuration of the system is then given by the set $(\mathbf{n}, \boldsymbol{\tau})=\left\{\left(n_{i}, \tau_{i}\right)\right\}_{i=1}^{L}$. The hopping rates out of site $i$ are taken to depend both on the occupation of the site $n_{i}$ and on the state of the clocks $\tau_{i}$, but not on the occupation of any other site, in accordance with the general approach of the ZRP.

Here we consider the following dynamics: particles hop between sites with rate $u(n, \tau)$. The internal clock on the target site is reset to zero together with a jump. Independently of the jump processes, at each site the internal clock is incremented by one unit with a constant rate $c$. For a jump from $i$ to $j$, these two processes can be schematically summarized by

$$
\begin{gathered}
\left(n_{i}, \tau_{i}\right),\left(n_{j}, \tau_{j}\right) \stackrel{u\left(n_{i}, \tau_{i}\right)}{\longrightarrow}\left(n_{i}-1, \tau_{i}\right),\left(n_{j}+1, \tau_{j}=0\right) \\
\left(n_{i}, \tau_{i}\right) \stackrel{c}{\longrightarrow}\left(n_{i}, \tau_{i}+1\right),
\end{gathered}
$$

The particle jump process, when taken by itself, is nonMarkovian, since the rate of a jump depends on how much time has passed since a particle last hopped into the jump site. Choosing the target site $j \neq i$ uniformly (i.e., consid- 
ering a fully connected graph) corresponds to mean-field (MF) dynamics, while restricting the target site to $j=i+$ 1 corresponds to totally asymmetric nearest-neighbor hopping on a ring.

Notice that the full process of particle jumps and clock increments together defines a Markovian process. Therefore, the model may be implemented by a discretetime Monte Carlo version of these dynamics with random sequential update which is defined as follows: let $p_{\max }=$ $\max _{n, \tau}[u(n, \tau)+c]$. For the Monte Carlo update pick a pair of sites $(i, j)$ uniformly and attempt to make one of the following changes: (i) move a particle to the target site $j$ with probability $u\left(n_{i}, \tau_{i}\right) / p_{\max }$ and reset the clock on the target site $j$ to zero, or, (ii) increment the internal clock on the starting site $i$ with probability $c / p_{\max }$. A total of $L$ consecutive updates constitute one Monte Carlo sweep.

Let us first consider the MF dynamics, whereby the target site $j$ is chosen uniformly. Since the hopping is to an arbitrary site, no correlation between sites is generated, and so the steady-state distribution factorizes into a product of single-site terms in the thermodynamic limit. The single-site occupation and clock probability in the steady state, $P(n, \tau)$, can then be found by examining a single site with a "mean-field" incoming current $J$ generated by all other sites. This is equivalent to a solution of a single site in the grand-canonical ensemble. The master equation for the single-site probability is

$$
\begin{aligned}
\frac{d P(n, \tau)}{d t}= & -P(n, \tau)[J+c+u(n, \tau)]+J P(n-1) \delta_{\tau, 0} \\
& +c P(n, \tau-1)+u(n+1, \tau) P(n+1, \tau),
\end{aligned}
$$

where the single-site marginal distribution is defined by $P(n) \equiv \sum_{\tau} P(n, \tau)$, and $J=\sum_{n, \tau} u(n, \tau) P(n, \tau)$ is the incoming current. These equations are valid also for $n=0$ and $\tau=0$ by defining $P(-1, \tau)=P(n,-1)=0$ and $u(0, \tau)=0$.

In the steady state, where $d P(n, \tau) / d t=0$, one obtains by summing Eqs. (3) over $\tau$

$$
\bar{u}(n) P(n)=J P(n-1) .
$$

Here $\bar{u}(n)$ is the mean hopping rate out of a site with $n$ particles

$$
\bar{u}(n) \equiv \frac{\sum_{\tau} P(n, \tau) u(n, \tau)}{\sum_{\tau} P(n, \tau)} .
$$

Equation (4) expresses the balance between the probabilities to hop into and out of a site with $n$ particles. The marginal distribution $P(n)$ is therefore the same as that of a Markovian ZRP [12], but with an effective hopping rate $\bar{u}(n)$. This gives the steady-state distribution

$$
P(n)=P(0) J^{n} \bar{f}(n) \quad \text { with } \quad \bar{f}(n)=\prod_{i=1}^{n} \bar{u}(i)^{-1} .
$$

Here $P(0)^{-1} \equiv 1+\sum_{n=1}^{\infty} J^{n} \bar{f}(n)$ ensures proper normalization of $P(n)$. The occurrence of condensation is determined by the asymptotic behavior of $P(n)$. For $P(n) \sim n^{-\alpha}$ condensation takes place for $\alpha>2$ [10]. In the Markovian case, ones has $\alpha=b$ for hopping rates of the form (1).

To analyze condensation in the non-Markovian case we proceed by considering, for simplicity, hopping rates of the form

$$
u(n, \tau)=\left\{\begin{array}{lll}
0 & \tau=0 & \text { ("off state") } \\
u(n) & \tau \geq 1 & \text { ("on state") }
\end{array}\right.
$$

In this case, whenever a particle hops into a site the site is switched to an "off" state in which no particles can hop out. Only after its clock reaches $\tau=1$ the site is turned "on" again, in which case particles hop out with a rate $u(n)$. This special case will be called the on-off model. In this case, the clock has in effect only two states: $\tau=0$ corresponding to off, and $\tau \geq 1$ corresponding to on. Correspondingly, the state of the site can be characterized by $P_{\text {off }}(n)=P(n, 0)$ and $P_{\text {on }}(n)=\sum_{\tau \geq 1} P(n, \tau)$.

The master equation for the stationary probability distribution (3) is then

$$
\begin{aligned}
P_{\mathrm{off}}(n)[J+c] & =J P(n-1), \\
P_{\mathrm{on}}(n)[J+u(n)] & =c P_{\mathrm{off}}(n)+P_{\mathrm{on}}(n+1) u(n+1) .
\end{aligned}
$$

First we study the probability $P_{\text {off }}$ to find a site in the off state. By summing over $n$, Eq. (8a) yields

$$
P_{\text {off }}=\frac{J}{c+J} .
$$

Further, from Eqs. (4), (8a), and (9) we find

$$
P_{\text {off }}(n)=\frac{\bar{u}(n) P(n)}{J+c}=\frac{P_{\text {off }}}{J} \bar{u}(n) P(n) .
$$

In addition, for $u(n, \tau)$ of the form (7), Eq. (5) gives

$$
P_{\text {on }}(n)=\frac{\bar{u}(n)}{u(n)} P(n) .
$$

The occurrence of condensation depends only on the asymptotic behavior of the effective hopping rate, $\bar{u}(n)$. This rate follows from (10) and (11) using $P_{\text {off }}(n)+$ $P_{\text {on }}(n)=P(n)$, and is given by

$$
\frac{1}{\bar{u}(n)}=\frac{P_{\text {off }}}{J}+\frac{1}{u(n)} .
$$

This states that the mean time between hops from a site with $n$ particles is equal to the mean time this site is in an off state plus the time it takes a particle to hop out once the system is already on. The current can be computed selfconsistently from $J=\sum_{n} \bar{u}(n) P(n)$.

In the case of $u(n)$ of the form (1), it can be seen from (12) that to leading order in $1 / n$ the effective hopping rates are again of the form (1), given by 


$$
\bar{u}(n) \sim \frac{c+J}{c+J+1}\left(1+\frac{b_{\mathrm{eff}}}{n}\right),
$$

with

$$
b_{\mathrm{eff}}=\frac{c+J}{c+J+1} b<b .
$$

From (6), we then have

$$
P(n) \sim\left[\frac{J(J+c+1)}{J+c}\right]^{n} n^{-b_{\mathrm{eff}} .}
$$

To analyze the condensation transition we note that for $J(J+c+1) /(J+c)<1$, the distribution decays exponentially with $n$ and the system is in a subcritical homogeneous phase. For a critical current given by $J_{c}=\left(c+J_{c}\right) /\left(c+J_{c}+1\right)$, the decay is algebraic, and one has condensation for $b_{\text {eff }}>2$. For the critical current we find

$$
J_{c}=\frac{c}{2}\left(\sqrt{1+\frac{4}{c}}-1\right)
$$

which allows us to write

$$
b_{\text {eff }}=J_{c} b .
$$

Therefore, condensation takes place for hopping parameter $b>\frac{4}{c}(\sqrt{1+4 / c}-1)^{-1}$ which is larger than 2 , in contrast with the Markovian case for which the critical value for condensation is $b=2$. This means that for densities $\rho=$ $N / L$ above a critical density $\rho_{c}$, the average number of particles in the condensate is $N_{\text {cond }}=L\left(\rho-\rho_{c}\right)$. All other sites have an average "background" density $\rho_{c}$.

We now turn to a ring geometry with nearest-neighbor hopping and fully asymmetric dynamics. In this case, the stationary distribution does not factorize. Simulations in the condensed phase of the on-off model with $u(n)$ given by Eq. (1) indicate that the condensate drifts with a finite velocity. In addition, we find that in contrast to previously known condensation phenomena, the condensate typically occupies two adjacent sites $i$ and $i+1$. These observations can be explained by a more detailed microscopic investigation of the dynamics. It turns out that the drift takes place via a "slinky" motion where particles hop from site $i$ to $i+1$ at a rate $u\left(n_{i}\right)$ which is approximately constant at large $n_{i}$, leaving site $i+1$ in a predominantly off-state. Thus particles accumulate at site $i+1$ until site $i$ is emptied, giving the clock at $i+1$ the chance to reach the onstate for durations of time sufficiently long to allow particles to escape. Then particles start to hop from site $i+1$ to site $i+2$ in the same fashion.

This mechanism suggests that the drift velocity $v_{\text {cond }}$ is inversely proportional to the number of particles in the condensate $N_{\text {cond }}$, i.e.,

$$
v_{\text {cond }}^{-1} \sim N-N_{c}=L\left(\rho-\rho_{c}\right) .
$$

In the thermodynamic limit, the velocity of the condensate vanishes. Superimposed on this motion, the condensate can melt and reappear at some other site of the lattice, similar to what happens in the Markovian case. This happens on a characteristic time which scales with the system size to a power larger than 2 [13-15].

In Fig. 1 we present snapshots at different times of the on-off model with totally asymmetric nearest-neighbor hopping for $L=1000$. One clearly sees that the condensate occupies two adjacent sites with varying relative occupation of the two sites, consistent with the slinky motion described above. The drift of the condensate is evident in the figure. In order to demonstrate the slinky motion in more detail, we present in Fig. 2 a plot showing the position of the most occupied site $i_{\max }$ and its occupation number, $n_{\max }$, as a function of time. The occupation number $n_{\max }$ oscillates in time with approximately constant frequency. Typically it decreases linearly until it reaches its minimal value, when $i_{\max }$ increases by 1 and $n_{\max }$ starts increasing.

In Fig. 3 we present the single-site occupation probability distribution $P(n)$ for various densities. At high densities the distribution exhibits a plateau which reflects the particle distribution among the two sites which constitute the condensate. This is in contrast with a Markovian ZRP where the condensate is supported by a single site, which results in a sharp peak in $P(n)$. The value of $P(n)$ at the plateau in the non-Markovian case may be estimated for $\rho$ above the critical density and large $L$ using the slinky motion of the condensate. The probability that a given site carries the condensate is $2 / L$, and in such a site there is an approximately uniform probability to find any occupation $0<n<N_{\text {cond }}=L\left(\rho-\rho_{c}\right)$. Thus,

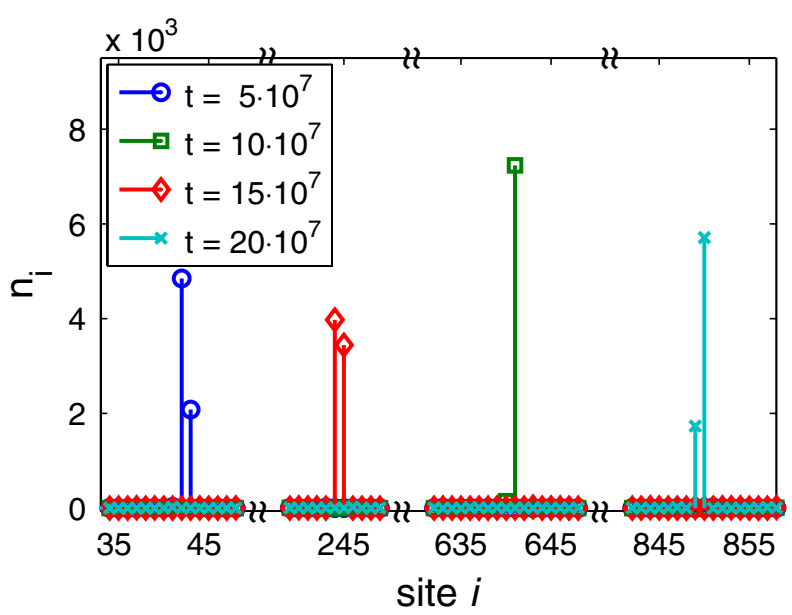

FIG. 1 (color online). Snapshots of the on-off model with totally asymmetric nearest-neighbor hopping on a ring, showing the occupation numbers $n_{i}$ at and in the vicinity of the condensate at four points in time. Here, $L=1000$ sites, $\rho=10, b=$ 5.5 , and $c=1$. The condensate occupies two sites and drifts with a constant mean velocity. 

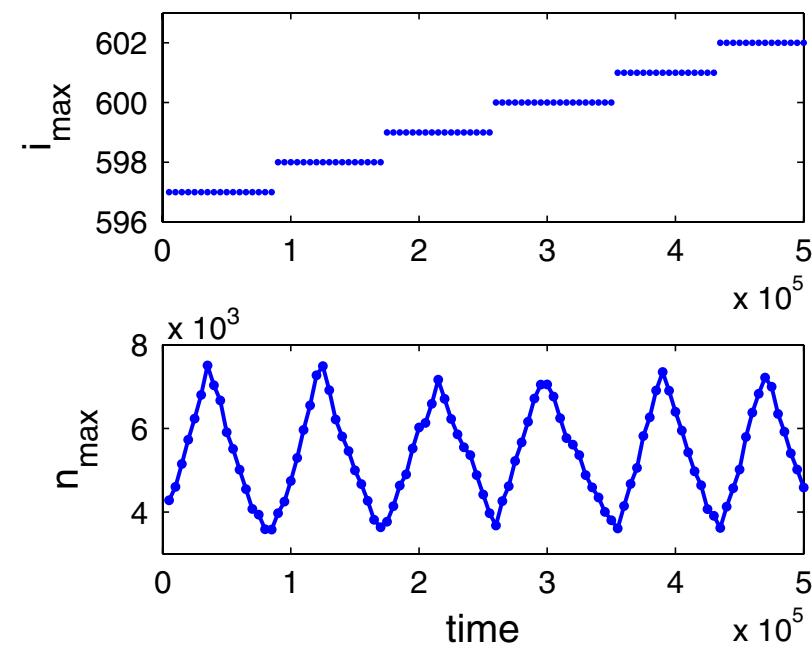

FIG. 2 (color online). The position of the most occupied site $i_{\max }$ and its occupation number $n_{\max }$ as a function of time, on a ring of size $L=1000$ with totally asymmetric nearest-neighbor hopping. Simulation parameters: $\rho=10, b=5.5$, and $c=1$.

$$
P_{\text {plateau }} \sim \frac{2}{L} \frac{1}{L\left(\rho-\rho_{c}\right)} .
$$

This estimate agrees well with the plateau value in Fig. 3. For small densities, $P(n)$ decays exponentially, indicating the absence of a condensate. For the system size studied in this figure, the distribution at small values of $n$ does not allow to extract a power law decay as expected for the

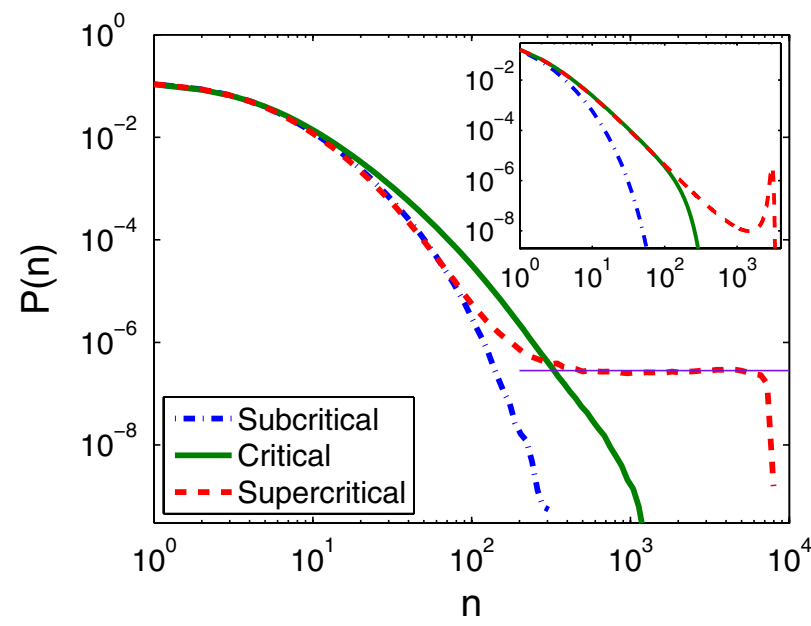

FIG. 3 (color online). The occupation probability $P(n)$ of a single-site in a ring with totally-asymmetric nearest-neighbor hopping and different densities, as obtained from Monte Carlo simulations. Here $L=1000$, and curves for subcritical density ( $\rho=3)$, supercritical density $(\rho=10)$ and at the critical region $(\rho=4.1)$ are presented. The horizontal line indicates $P_{\text {plateau }}$ of (19), where $\rho_{c}$ was obtained from the simulation. The inset shows a similar plot of $P(n)$ for a Markovian ZRP of $L=$ 1000 sites with $b=5$, in the subcritical $(\rho=0.5)$, critical $(\rho=$ $1)$, and supercritical $(\rho=4)$ phases. condensation transition. At density $\rho=4.1$ there is a range of $n$ for which $P(n)$ seems to follow a power law with $b_{\text {eff }} \approx 4$. This value differs significantly from $b=5.5$, the expected value for Markovian ZRP.

So far, we discussed in detail a simple on-off model which has been used to demonstrate the effect of nonMarkovian dynamics on the steady-state distribution and on condensation. For MF dynamics, the asymptotic behavior of the effective rates can be obtained for the more general class of models with rates of the form $u(n, \tau)=$ $u(n) v(\tau)$. Generally, $b_{\text {eff }}$ may be larger or smaller than the "bare" value of $b$. Interestingly, one can introduce an onoff model with nearest-neighbor hopping for which the stationary distribution factorizes strictly even on finite lattices and where the effective hopping rates can be computed exactly. In this model particles jump with rates (7) but the advancement of the clock depends on the clock states of neighboring sites [16].

The findings of this work suggest that the temporal correlations may significantly alter the condensation transition and the nature of the condensate in general driven systems. While this result is based on studies of a particular dynamical process, we believe it is rather generic and applies to a wide class of temporally correlated processes.

We thank M. R. Evans for useful comments. The support of the Israel Science Foundation (ISF) and the Albert Einstein Minerva Center for Theoretical Physics is gratefully acknowledged. This work was carried out in part while G. M. S. was at the Weizmann Institute of Science.

[1] Y. Kafri, E. Levine, D. Mukamel, G. M. Schütz, and J. Török, Phys. Rev. Lett. 89, 035702 (2002).

[2] Y. Kafri, E. Levine, D. Mukamel, G. M. Schütz, and R. D. Willmann, Phys. Rev. E 68, 035101(R) (2003).

[3] E. Levine, G. Ziv, L. Gray, and D. Mukamel, Physica (Amsterdam) 340A, 636 (2004).

[4] J. Kaupužs, R. Mahnke, and R. J. Harris, Phys. Rev. E 72, 056125 (2005).

[5] A. G. Angel, M. R. Evans, E. Levine, and D. Mukamel, Phys. Rev. E 72, 046132 (2005).

[6] S. N. Dorogovtsev and J.F.F. Mendes, Evolution of Networks (Oxford University Press, Oxford, 2003).

[7] K. van der Weele, D. van der Meer, M. Versluis, and D. Lohse, Europhys. Lett. 53, 328 (2001).

[8] J. Török, Physica (Amsterdam) 355A, 374 (2005).

[9] F. Spitzer, Adv. Math. 5, 246 (1970).

[10] M. R. Evans and T. Hanney, J. Phys. A 38, R195 (2005).

[11] S. Chatterjee and M. Barma, Phys. Rev. E 77, 061124 (2008).

[12] E. D. Andjel, Ann. Probab. 10, 525 (1982).

[13] S. Grosskinsky, G. M. Schütz, and H. Spohn, J. Stat. Phys. 113, 389 (2003).

[14] C. Godrèche and J. M. Luck, J. Phys. A 38, 7215 (2005).

[15] J. Beltran and C. Landim, arXiv:0802.2171v1.

[16] O. Hirschberg, D. Mukamel, and G. M. Schütz (to be published). 